\title{
Partial Seizures as the First Manifestation of Primary Angiitis of the Central Nervous System
}

\author{
Elisa Brauns $^{1}$, Sophie Schuind ${ }^{2}$, Laetitia Lebrun ${ }^{3}$, Valentine Inthasot ${ }^{4}$, Frédéric Vandergheynst ${ }^{5}$ \\ ${ }^{1}$ Service de Médecine Interne, CHU Erasme, Brussels, Belgium \\ ${ }^{2}$ Service de Neurochirurgie, CHU Erasme, Brussels, Belgium \\ ${ }^{3}$ Service d'Anatomo-pathologie, CHU Erasme, Brussels, Belgium \\ ${ }^{4}$ Service de Médecine Interne, Hôpital d'Ixelles, Brussels, Belgium \\ ${ }^{5}$ Service de Médecine Interne, CHU Erasme, Brussels, Belgium
}

Received: $12 / 11 / 2017$

Accepted: 20/11/2017

Published: $27 / 12 / 2017$

How to cite this article: Brauns E, Schuind S, Lebrun L, Inthasot V, Vandergheynst F. Partial seizures as the first manifestation of primary angiitis of the central nervous system. EJCRIM 2017;4: doi:10.12890/2017_000784.

Conflicts of Interests: The Authors declare that there are no competing interests.

This article is licensed under a Commons Attribution Non-Commercial 4.0 License

\section{ABSTRACT}

A young woman presented with initial epileptic seizures and multiple 'mass-like' lesions seen on computed tomography and magnetic resonance imaging. Given this presentation and her past medical history, a cerebral biopsy was performed to rule out malignancy, but showed a necrotizing cerebral vasculitis. We describe this case to reinforce the view that primary central nervous system vasculitis (PCNSV) should be considered in the differential diagnosis of a cerebral mass, even if uncommon.

\section{LEARNING POINTS}

- PCNSV may cause a variety of clinical manifestations including seizures.

- PCNSV lesions can mimic a cerebral mass.

- Brain biopsy remains the gold standard for the diagnosis of PCNSV, especially to rule out a cancer diagnosis.

\section{KEYWORDS}

Cerebral angiitis, seizure, primary central nervous system vasculitis

\section{CASE DESCRIPTION}

A 43-year-old woman was admitted to hospital with hypertonia of the right leg, then of the right arm and finally of the right side of the face. She also presented with dysarthria and apraxia. There was no evidence of tongue biting and no urinary incontinence was reported. A CT scan of the brain without contrast showed hypodensity in the left hemisphere with mass effect. She was transferred to our institution for further investigation. Cerebral CT angiography showed one lesion on the left side of the cerebral falx with severe edema causing a mass effect on the left lateral ventricle; and also a second lesion of centimetric hypodensity in front of the frontal horn of the left ventricle, without enhancement after contrast injection. She had no significant history except for the removal of precancerous skin lesions, followed up annually in dermatology. She had also been screened for colorectal and breast cancer the year before. She was not taking any medication, never smoked, and was a sports teacher by profession. She reported no headaches, weight loss, arthralgia or any other systemic symptoms. 
Blood analysis showed unremarkable hematological and inflammatory parameters (C-reactive protein and erythrocyte sedimentation rate) and normal liver and kidney functions; the only abnormality was D-dimer level elevated at $5567 \mathrm{ng} / \mathrm{mL}(\mathrm{N}<400 \mathrm{ng} / \mathrm{mL})$.

She was hospitalized in the internal medicine department and treated with corticosteroids (methylprednisolone $32 \mathrm{mg}$ three times daily) and levetiracetam (500 mg twice daily). An MRI scan showed multiple bilateral supratentorial lesions, some presenting with a spontaneous T1 hypersignal and all with enhancement after gadolinium injection. The two most voluminous lesions $(15 \times 10 \times 19 \mathrm{~mm}$ and $8 \times 4 \mathrm{~mm})$ appeared as target lesions, with severe edema. A meningeal gadolinium enhancement was also described (Fig. 1). The first hypothesis, based on her history of precancerous skin lesions and the aspect on imaging, was of metastatic melanoma. Biopsy of a suspicious cutaneous dorsal lesion however turned out to be negative. A 18-fluorodeoxyglucose positron emission tomography CT (18FDG-PET-CT) scan demonstrated no remarkable hypermetabolic areas, including brain masses.

The patient was subsequently readmitted to the emergency department for a recurrence of partial seizures, for which the dosage of levetiracetam was increased. A C-11 methionine PET-CT scan showed slight hypermetabolism in one of the left frontal lesions, with no typical features of brain metastases or glioblastoma. Given the lack of a definitive diagnosis, excision of the prefrontal parasagittal mass was performed using neuronavigation. The ablation appeared macroscopically complete.

Postoperatively, the patient experienced a worsening of her right hemiparesis, predominantly in the lower limb. The dosage of methylprednisolone was however decreased to $32 \mathrm{mg}$ daily, and physiotherapy was commenced.

Surgical pathology revealed polymorphic lymphoplasmacytic perivascular infiltrate with focal vascular thrombosis and related ischemic necrosis of the parenchyma. There were no granuloma, no signs of demyelination or malignancy, and no evidence of beta-amyloid vascular deposition or viral infection (immunolabelling was negative for CMV and HSV). The diagnosis of necrotizing vasculitis was proposed by pathologists (Fig. 2). Regarding autoimmune and infectious diseases, serologies for antinuclear antibodies, antineutrophil cytoplasmic antibodies, rheumatoid factor, and HIV/hepatitis/syphilis were negative.

The patient received intravenous pulses of $1000 \mathrm{mg}$ of cyclophosphamide (CYC - $12.5 \mathrm{mg} / \mathrm{kg}$ ), every 2 weeks for the three first doses and then every 3 weeks. Oral methylprednisolone was progressively decreased to $4 \mathrm{mg}$ per day. During the treatment, the patient experienced a deep venous thrombosis; hence, anticoagulation by acenocoumarol was introduced. After administration of six doses of CYC, magnetic resonance angiography showed an improvement of the edema and a reduction in size of most of the previously enhanced lesions, without abnormalities in the main cerebral arteries (Fig. 3). CYC was discontinued and mycophenolate mofetil started, while the same dose of oral methylprednisolone was continued. The patient's right hemiparesis persists, mainly of the lower limb, for which rehabilitation therapy is ongoing.
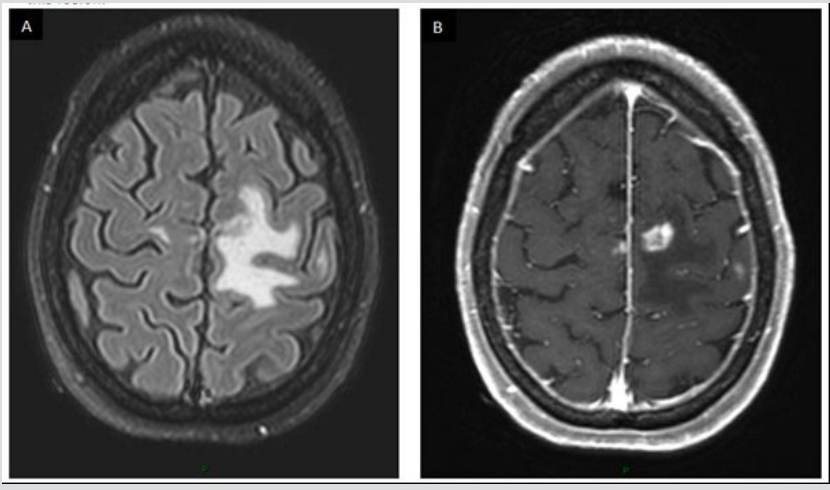

Figure 1. MRI scan of the brain using fluid-attenuated inversion recovery (FLAIR) sequence showing (A) a left paracentral lesion of $20 \mathrm{~mm}$ in diameter, surrounded by severe edema and $\mathrm{T} 1$ sequence after gadolinium injection showing $(B)$ an enhancement of this lesion

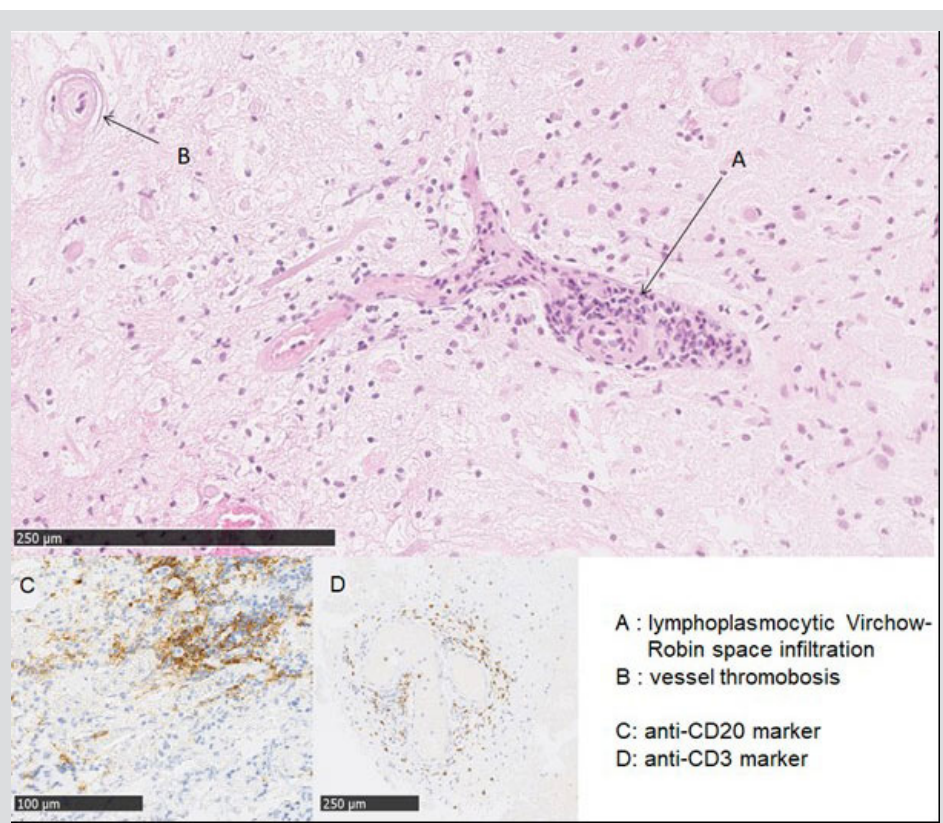

Figure 2. Surgical pathology showing (A) lymphoplasmacytic infiltration of Virchow-Robin spaces; (B) vessel thrombosis; (C) anti-CD20 marker; (D) anti-CD3 marker 


\section{DISCUSSION}

Our case illustrates a rare presentation of an uncommon disease, PCNSV, with an annual incidence rate reported of 2.4 cases per 1,000,000 person-years ${ }^{[1]}$. It affects small and medium-sized blood vessels of the brain and, less frequently, of the spinal cord, without evidence of systemic involvement. Clinical manifestations can vary, with focal deficits or more nonspecific symptoms (headache and cognitive impairment). Seizures occur in about $15 \%$ of cases $^{[2]}$. MRI is abnormal in most patients, generally with gadolinium enhancement of the lesions. Presentation with a mass lesion accounts for less than $5 \%$ of cases ${ }^{[3]}$. Angiography usually reveals vessel abnormalities; however, these lesions can be seen in many other conditions such as atherosclerosis and cerebral vasospasm ${ }^{[4]}$. Cerebrospinal fluid analysis is generally pathologic but nonspecific.

Only a brain biopsy can provide a definite diagnosis, with increased sensitivity when targeting a radiological lesion, as with our patient. Inclusion of leptomeninges also raises the biopsy sensitivity ${ }^{[5]}$. Three histological patterns can be seen in order of frequency: granulomatous, lymphocytic, and necrotizing; however, this classification appears to have no impact on the radiological pattern. There are no established guidelines for the treatment, its duration and options in the event of relapse. We have used the most common treatment regimen described in the literature, consisting of a combination of cyclophosphamide and corticosteroids.

In conclusion, the originality of this case lies in the combination of two atypical presentations of PCNSV: mass-like lesions from a radiological point of view and seizures from a clinical point of view.

\section{REFERENCES}

1. Salvarani C, Brown RD, Christianson TJ, Miller DV, Giannini C, Huston J, et al. An update of the Mayo Clinic cohort of patients with adult primary central nervous system vasculitis: description of 163 patients. Medicine 2015;94:e738.

2. Salvarani C, Brown RD, Hunder GG. Adult primary central nervous system vasculitis. Lancet 2012;380:767-77.

3. Molloy ES, Singhal AB, Calabrese LH. Tumour-like mass lesion: an under-recognised presentation of primary angiitis of the central nervous system. Ann Rheum Dis 2008;67:173235.

4. Kadkhodayan Y, Alreshaid A, Moran CJ, Cross DT, Powers WJ, Derdeyn CP. Primary angiitis of the central nervous system at conventional angiography. Radiology 2004;233:87882.

5. Miller DV, Salvarani C, Hunder GG, Brown RD, Parisi JE, Christianson TJ, et al. Biopsy findings in primary angiitis of the central nervous system. Am J Surg Pathol 2009;33:35-43. 